\title{
Positron emission tomography in cases of chorea with different underlying diseases
}

\author{
SHINICHI HOSOKAWA,* YUICHI ICHIYA, $\dagger$ YASUO KUWABARA, $\dagger$ \\ ZENJI AYABE, $\dagger$ KUNIHIKO MITSUO, $\ddagger$ IKUO GOTO, $\ddagger$ MOTOHIRO KATO,* \\ From the Departments of Neurophysiology,* Radiology, $\dagger$ and Neurology, $\ddagger$ Faculty of Medicine, Kyushu \\ University, Fukuoka, Japan
}

SUMMARY Local cerebral metabolic rate for glucose (LCMRglc) was measured with positron emission tomography using the ${ }^{18} \mathrm{~F}$-fluorodeoxy-glucose method in five patients with chorea due to different underlying diseases. Hypometabolism was observed in the striatum bilaterally in patients with Huntington's disease, choreoacanthocytosis, sporadic progressive chorea and dementia, and pseudo-Huntington form of dentato-rubro-pallido-luysian atrophy (DRPLA). The patient with hemichorea showed hypometabolism in the striatum on the contralateral side to the chorea. The patient with pseudo-Huntington form of DRPLA showed a diffusely decreased LCMRglc in other structures including the cerebral cortex, thalamus and cerebellum. These findings indicated that dysfunction of the striatum is relevant to the genesis of chorea in all these patients, even though the extent of dysfunction in other structures is different in each case.

Chorea may appear as a symptom of a wide variety of degenerative, vascular, metabolic or toxic disorders involving the central nervous system. Although dysfunction of the basal ganglia, particularly of the striatum, is generally assumed to be responsible, the precise pathophysiological mechanism giving rise to choreic movements has yet to be determined. Recent studies ${ }^{12}$ have shown that positron emission tomography (PET), for the measurement of local cerebral metabolism, is a useful tool to elucidate the dysfunction of basal ganglia in Huntington's disease patients and in subjects at risk for that disease. In the present study, we have examined five cases of chorea associated with different underlying diseases, using PET to obtain some insight into the pathophysiological mechanism of chorea.

\section{Case reports}

Patient 1 This 39 year old man noted involuntary movements in his tongue and limbs at age 34 . His family noticed character changes such as emotional irritability, at the same

Address for reprint requests: Shinichi Hosokawa, M.D., Departmen of Neurophysiology, Neurological Institute, Faculty of Medicine, Kyushu University 60, Higashi-ku, Fukuoka 812, Japan.

Received 5 September 1986. Accepted 6 November 1986. time. His father and his grandmother had a similar disease. On admission, he had mental impairment, vertical gaze paresis, chorea in his tongue, perioral muscle and distal limb muscles, and the Babinski sign was positive bilaterally. His deep tendon reflexes were generally increased. The brain computed tomography $(\mathrm{CT})$ revealed moderate dilatation of the anterior horn of the lateral ventricles (fig C). He was diagnosed as having Huntington's disease.

Patient 2 This 32 year old woman had difficulty in swallowing since age 29. She had had several attacks of loss of consciousness, and had noted choreic involuntary movement in the limbs. There was no family history of neurological diseases. At age 32, she had normal intelligence, oro-facial dyskinesia with tongue biting, and choreic movements of the neck, shoulder and distal limb muscles. The deep tendon reflexes were all absent. The laboratory data showed increased serum creatine kinase and numerous acanthocytes in the blood. Brain CT showed mild dilatation of the anterior horn of the lateral ventricles (fig e). She was diagnosed as having choreoacanthocytosis. ${ }^{3}$

Patient 3 This 53 year old woman had worked as a nurse and had been in normal health until age 49 when she became forgetful. From the age of 51 , she also noted involuntary movement of all four limbs. Her family was reported to be free of neurological disease. At age 53, she had mild dementia, dystonic posture of the trunk and choreic movements of the neck and distal limb muscles. Brain CT showed mild dilatation of the anterior horn of the lateral ventricles (fig g). She was suspected of having Huntington's disease. However, autosomal dominant inheritance could not be found.

Patient 4 This 73 year old woman was noticed to have 

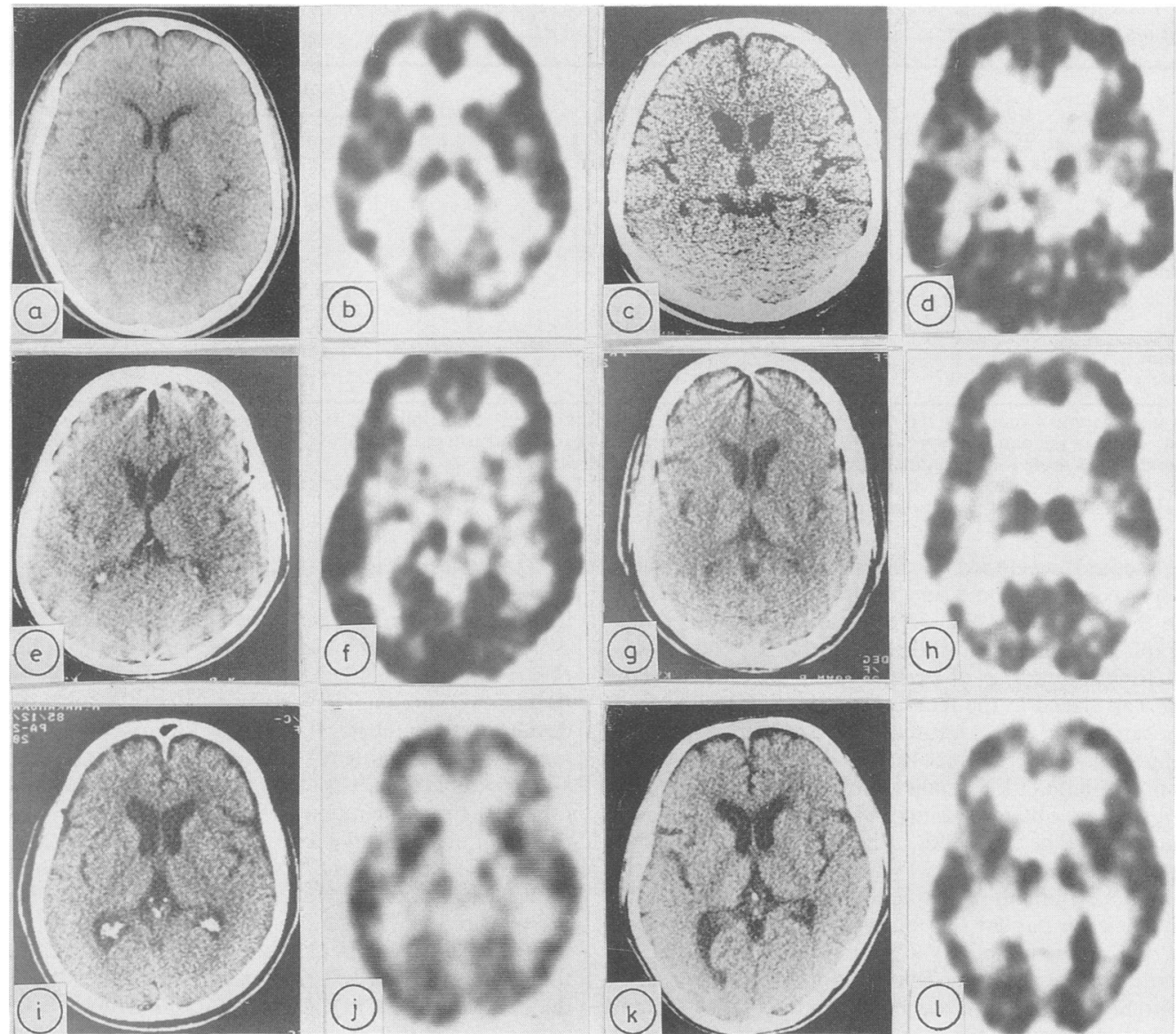

Fig The brain CT and PET images reflecting LCMRglc at the level of the striatum in healthy control $(a, b)$, patient 1 (Huntington's disease) $(c, d)$, patient 2 (choreoacanthocytosis) $(e, f)$, patient 3 (sporadic chorea) $(g, h)$, patient 4 (hemichorea) $(i, j)$, and patient 5 (pseudo-Huntington's form of DRPLA) $(k, l)$. The right hemisphere is on the right hand sides of both CT and PET images. Patient 1 shows moderate dilatation of the anterior horn of the lateral ventricles $(c)$, and marked hypometabolism of the striatum $(d)$. Patients 2 and 3 show relatively mild dilatation of the anterior horn of the lateral ventricles $(e, g)$ and marked hypometabolism of the striatum $(f, h)$. The $C T$ in patient 4 shows no asymmetry (i), but PET shows decreased LCMRglc of the right caudate nucleus, as compared with the left side $(j)$. Patient 5 shows mild dilatation of the lateral ventricles $(k)$, but the pattern of LCMRglc is normal ( $l$ ).

choreic involuntary movement in her left arm when aged $\mathbf{4 0 .}$ This involuntary movement had not progressed. At age 73, she had brisk, choreic involuntary movement in her left shoulder, arm and chest. Needle EMG revealed irregular appearance of bursts of motor unit discharges with a duration of $200-300 \mathrm{~ms}$ and an interval of $100-300 \mathrm{~ms}$, which was consistent with chorea. Brain CT (fig I) and MRI showed a mild diffuse dilatation of the lateral ventricles, but showed no asymmetry. She was diagnosed as having hemichorea probably of vascular origin.

Patient 5 This 28 year old man had progressive mental deterioration and gait disturbance, and several episodes of generalised convulsion and unconsciousness since the age of
28 years. He noted the involuntary movement of limbs at age 31. His paternal granduncle and uncle were reported to have a similar neurological disease. At age 34, he had dementia, jerky eye movement, oral dyskinesia, choreic movement of limbs, limb ataxia, and wide-based gait. His brain CT showed a mild diffuse dilatation of the lateral ventricles (fig k) and moderate cerebellar atrophy. He was diagnosed as having pseudo-Huntington form of dentato-rubro-pallidoluysian atrophy (DRPLA).

\section{PET studies}

PET was performed in three healthy volunteers (33-37 yearold males) and the five patients, using the ${ }^{18} \mathrm{~F}$-fluorodeoxy- 
Table Local cerebral metabolic rate for glucose ( LCMRglc) $(\mathrm{mg} / 100 \mathrm{ml} / \mathrm{min})$ in healthy control and patients of chorea with different underlying diseases $\dagger$

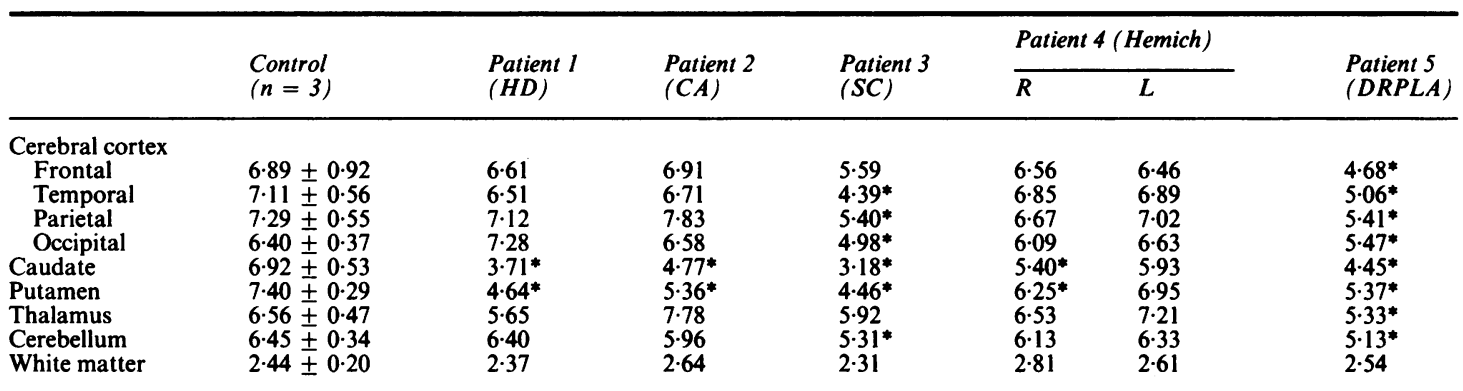

*Values in the control represent the mean \pm SD of LCMRglc $(\mathrm{mg} / 100 \mathrm{cc} / \mathrm{min})$ of the bilateral sides. Since no significant asymmetry was observed, values in the patients represent the means of the bilateral sides except those in patient 4 where the values of each side are represented. *Decreased values more than 2 standard deviations from the mean control values.

HD, Huntington's disease; CA, choreoacanthocytosis; SC, sporadic progressive chorea and dementia; Hemich, hemichorea; DRPLA, pseudo-Huntington form of dentato-rubro-pallido-luysian atrophy.

glucose method developed by Phelps et al. ${ }^{4}$ The subjects were deprived of food and any medication for at least $5 \mathrm{~h}$ prior to the PET study, and were required to lie still in the tomograph in a quiet semi-darkened room. No patient was sedated for the purpose of performing the PET study. A catheter was placed in the femoral artery to obtain arterial blood samples. We infused 3 to $10 \mathrm{mCi}$ of $\left[{ }^{18} \mathrm{~F}\right] 2$-fluoro-2-deoxy-D-glucose (FDG) into the antecubital vein for 1 minute. PET images of local cerebral glucose metabolism were obtained using SET-130W (HEADTOME III, Shimadzu). The full width at half maximum (FWHM) measure of spatial resolution was $8.2 \mathrm{~mm}$ in the medial resolution mode. Attenuation correction was determined individually for each patient by obtaining a transmission scan using a ring source of activity $\left({ }^{68} \mathrm{Ge}\right)$. Scans were started 45 min after the FDG injection. Ten levels of images, parallel to the orbitomeatal line, were scanned at an interval of $7.5 \mathrm{~mm}$ from the lower level of the cerebellum to above the lateral ventricles. The values of local cerebral metabolic rate for glucose (LCMRglc) were calculated, according to the equation developed by Phelps et al. ${ }^{4}$ The LCMRglc was analysed both by visual observation of the pattern of PET images and by the quantitative analysis of LCMRglc values.

\section{Results}

\section{Visual inspection of PET images}

The visual inspection of PET images showed similar abnormalities in the patients with Huntington's disease (patient 1), choreoacanthocytosis (patient 2) and the patient with sporadic progressive chorea and dementia (sporadic chorea) (patient 3), as compared with the healthy controls. They showed apparent decreases in LCMRglc in the bilateral caudate nucleus and putamen (figs D, F and $\mathrm{H}$ ). No abnormality was observed throughout the rest of the brain including the cerebral cortex, thalamus and cerebellum in these patients. The hemichorea patient (patient 4) showed decreased LCMRglc in the caudate nucleus on the contralateral side of the chorea (fig j). No abnormality was observed in the pattern of PET images in the patient of pseudo-Huntington form of DRPLA (patient 5) in comparison with the healthy control (figl).

\section{Quantitative analysis of LCMRglc values}

The quantitative analysis of LCMRglc values confirmed the findings observed by visual inspection of PET images (table). No significant asymmetry of the LCMRglc values was observed in patients 1, 2, 3, and 5. The LCMRglc values in the caudate and putamen decreased more than two standard deviations from the normal means in the patients with Huntington's disease (patient 1), choreoacanthocytosis (patient 2) and sporadic chorea (patient 3). The LCMRglc values in other structures including the cerebral cortex were within the normal range (mean $\pm 2 \mathrm{SD}$ of healthy controls) in these patients except for the patient of sporadic chorea who showed decreased LCMRglc in the temporal, parietal, and occipital cerebral cortices, and the cerebellum. The patient with hemichorea (patient 4) showed decreased LCMRglc in the caudate nucleus and putamen on the side contralateral to the chorea. The LCMRglc values in the patient of DRPLA (patient 5) were diffusely low in all the gray matter structures including the cerebral cortex, caudate, putamen, thalamus and cerebellum.

\section{Discussion}

Since LCMRglc has been successfully used as an indicator of local functional brain activity, ${ }^{5}$ the findings in this study may be assumed to show local cerebral dysfunction in cases of chorea with different underlying diseases.

In the Huntington's disease patient (patient 1), bilateral hypometabolism in the caudate nucleus and putamen was observed, which confirmed the findings of previous studies by Kuhl et al ${ }^{1}$ and Martin et al. ${ }^{2}$ No significant abnormality in glucose metabolism was observed in other structures including the cerebral cortex or thalamus in this patient.

This paper is the first to report marked hypometabolism in both caudate nucleus and putamen in choreoacanthocytosis (patient 2). It has been shown that the patients with choreoacanthocytosis have 
atrophy of the caudate nucleus pathologically as well as on CT, and that there is neuronal loss in the striatum. ${ }^{6}$ The different characteristics of involuntary movement between patients with choreoacanthocytosis and those with Huntington's disease have been also documented electrophysiologically. ${ }^{7}$ The present findings indicate that dysfunction of the striatum is relevant in the genesis of chorea also in choreoacanthocytosis.

Although the underlying disease of patient 3 who showed progressive chorea and dementia without family history is unknown, the patient showed hypometabolism in the striatum, indicating the dysfunction of this structure as a cause of chorea. Moreover, hypometabolism in the cerebral cortices and cerebellum in this patient suggests wider involvements of the brain than in patients 1 and 2 .

It also should be noted that striatal hypometabolism was marked in patients 2 and 3 , even though the dilatation of the anterior horn of the lateral ventricle was relatively mild on CT.

The involuntary movement in patient 4 , involving the left shoulder and arm, was consistent with chorea as judged by the pattern of the movement and that of EMG discharges. ${ }^{8}$ Even though her brain CT showed no asymmetry, PET study demonstrated hypometabolism in the caudate nucleus and putamen on the side contralateral to the chorea.

Patient 5 was diagnosed clinically as having pseudo-Huntington form of DRPLA, ${ }^{9}$ because of positive family history, progressive ataxia, chorea and mental deterioration, and diffuse cerebral and cerebellar atrophy on CT. Histological studies have shown that there is no abnormality in the striatum in cases of pseudo-Huntington form of DRPLA. ${ }^{9}$ Even though no abnormality was observed in the pattern of PET images in patient 5 , hypometabolism in the striatum was demonstrated also in this patient by the quantitative analysis of LCMRglc. Moreover, his LCMRglc values were diffusely low in many structures including the cerebral cortex, thalamus and cerebellum, and which may be relevant to the diverse clinical manifestations in this patient such as dementia, ataxia, and epilepsy.
In summary, the common hypometabolism in the striatum in patients $1,2,3,4$, and 5 indicates that dysfunction of the striatum is relevant in the genesis of chorea in these patients, and that chorea may be caused by a common pathophysiological mechanism, even though their underlying diseases were different from each other. However, the extent of dysfunction in other structures was different in each case.

The authors thank Drs S Minato, K Tanaka, and S Tashima for their help. We also thank Dr S Mawatari (National Chikugo Hospital) for his cooperation.

\section{References}

1 Kuhl DE, Phelps ME, Maukham $\mathrm{CH}$, et al. Cerebral metabolism and atrophy in Huntington's disease determined by ${ }^{18} \mathrm{FDG}$ and computed tomographic scan. Ann Neurol 1982;12:425-34.

2 Martin WUW, Hayden MR, Suchowersky O, et al. Striatal metabolism in Huntington's disease and in benign hereditary chorea. Ann Neurol 1984;16:126.

3 Sakai T, Mawatari S, Iwashita H, et al. Coreoacanthocytosis. Clues to clinical diagnosis. Arch $\mathrm{Neu}$ rol 1981;38:335-8.

4 Phelps ME, Huang SC, Hoffman EJ, et al. Tomographic measurement of local cerebral glucose metabolic rate in humans with (F-18)2-fluoro-2-deoxy-D-glucose. Validation of method. Ann Neurol 1979;6:371-88.

5 Phelps ME, Mazziotta JC, Huang SC. Study of cerebral function with positron computed tomography. J Cereb Blood Flow Metabol 1982;2:113-62.

6 Bird TD, Cederbaum S, Valpey RW, et al. Familial degeneration of the basal ganglia with acanthocytosis: a clinical, neuropathological, and neurochemical study. Ann Neurol 1978;3:253-8.

7 Shibasaki H, Sakai T, Nishimura H, et al. Involuntary movements in chorea-acanthocytosis: a comparison with Huntington's chorea. Ann Neurol 1982;12:311-4.

8 Marsden CD, Obeso JS, Rothwell JC. Clinical neurophysiology of muscle jerks: myoclonus, chorea, and tics. In: Desmedt JE, ed. Motor Control Mechanisms in Health and Disease, New York: Raven Press, 1983:865-81.

9 Iizuka R, Hirayama K, Maehara K. Dentato-rubropallido-luysian atrophy: a clinico-pathological study. $J$ Neurol Neurosurg Psychiatry 1984;47:1288-98. 